\title{
Integration of Artificial Intelligence, Blockchain, and Wearable Technology for Chronic Disease Management: A New Paradigm in Smart Healthcare*
}

\author{
Yi XIE ${ }^{1,2 \dagger}$, Lin $\mathrm{LU}^{1,2 \dagger}$, Fei GAO ${ }^{1,2}$, Shuang-jiang $\mathrm{HE}^{2,3}$, Hui-juan $\mathrm{ZHAO}^{2,3}$, Ying FANG ${ }^{2}$, Jia-ming YANG ${ }^{2}$, Ying $\mathrm{AN}^{2,4}$, \\ Zhe-wei $\mathrm{YE}^{1,2}$, Zhe DONG ${ }^{5 \#}$ \\ ${ }^{I}$ Department of Orthopedic Surgery, Union Hospital, Tongji Medical College, Huazhong University of Science and Technology, \\ Wuhan 430022, China \\ ${ }^{2}$ Laboratory of Intelligent Medicine, Union Hospital, Tongji Medical College, Huazhong University of Science and Technology, \\ Wuhan 430022, China \\ ${ }^{3}$ School of Electronic Information and Communications, Huazhong University of Science and Technology, Wuhan 430074, \\ China \\ ${ }^{4}$ Wuhan Fourth Hospital, Tongji Medical College, Huazhong University of Science and Technology, Wuhan 430032, China \\ ${ }^{5}$ School of Cyber Science and Engineering, Huazhong University of Science and Technology, Wuhan 430074, China
}

(C) Huazhong University of Science and Technology 2021

\begin{abstract}
[Abstract] Chronic diseases are a growing concern worldwide, with nearly $25 \%$ of adults suffering from one or more chronic health conditions, thus placing a heavy burden on individuals, families, and healthcare systems. With the advent of the "Smart Healthcare" era, a series of cutting-edge technologies has brought new experiences to the management of chronic diseases. Among them, smart wearable technology not only helps people pursue a healthier lifestyle but also provides a continuous flow of healthcare data for disease diagnosis and treatment by actively recording physiological parameters and tracking the metabolic state. However, how to organize and analyze the data to achieve the ultimate goal of improving chronic disease management, in terms of quality of life, patient outcomes, and privacy protection, is an urgent issue that needs to be addressed. Artificial intelligence (AI) can provide intelligent suggestions by analyzing a patient's physiological data from wearable devices for the diagnosis and treatment of diseases. In addition, blockchain can improve healthcare services by authorizing decentralized data sharing, protecting the privacy of users, providing data empowerment, and ensuring the reliability of data management. Integrating AI, blockchain, and wearable technology could optimize the existing chronic disease management models, with a shift from a hospital-centered model to a patient-centered one. In this paper, we conceptually demonstrate a patient-centric technical framework based on AI, blockchain, and wearable technology and further explore the application of these integrated technologies in chronic disease management. Finally, the shortcomings of this new paradigm and future research directions are also discussed.
\end{abstract}

Key words: artificial intelligence; blockchain; wearable technology/devices; chronic diseases; smart healthcare; health monitoring; personalization; healthcare management; patient-centric

Nowadays, chronic disease is the dominant cause of morbidity and mortality worldwide, and the management of chronic diseases has become

Yi XIE, Email: 455085617@qq.com; Lin LU, Email: 1ledu2014@163.com

${ }^{\dagger}$ These authors contributed equally to this work and should be considered as co-first authors.

\#Corresponding author, E-mail: 13601706191@139.com

${ }^{*}$ This work was supported by the National Natural Science Foundation of China (No. 81974355 and No. 82172525), the National Intelligence Medical Clinical Research Center (No. 2020021105012440), and the Hubei Province Technology Innovation Major Special Project (No. 2018AAA067). an unavoidable issue in the home care industry due to the arrival of an aging society and the increase in the proportion of dual-income families ${ }^{[1-3]}$. The cost of treating traditional chronic diseases such as cardiovascular diseases in the United States was $\$ 555$ billion in 2014 and is expected to rise to $\$ 1.1$ trillion by $2035^{[2]}$. The common chronic diseases mainly include cancer, cardiovascular and cerebrovascular diseases, hypertension, chronic respiratory diseases, diabetes, obesity, chronic kidney diseases, degenerative diseases of joints, and neurodegenerative diseases ${ }^{[3]}$. With the extensive development of wearable devices, artificial intelligence (AI), and blockchain, the integration of these technologies can be applied to track the health 
status of patients suffering from chronic diseases.

In the management of chronic diseases, wearable devices can be worn directly on the body in the form of portable medical electronic equipment for sensing, recording, analyzing, regulating, and intervening to keep the person wearing the device healthy ${ }^{[4,5]}$. By conducting comprehensive, dynamic, and intelligent analysis of various health indicators, wearables enable the monitoring of users all day and provide personalized services for patients with chronic diseases. Based on these available multidimensional clinical and biological data sets, the implementation of high-performance computing and AI can predict risks more accurately ${ }^{[6]}$. By learning about new cases that have happened in the past, many AI algorithms are capable of catching related knowledge to enhance themselves. The AI-powered individualized treatment provides clinicians with an opportunity to specifically tailor early interventions to each patient with chronic diseases $^{[7]}$.

However, presently, mostmedical service platforms store patient data in their local central database, which may lead to asymmetry, opacity, and tampering of information. In addition, the data storage mode may lead to the lack of effective information exchange among medical units, resulting in the "information island phenomenon" thus reducing the automation level of machine learning in $\mathrm{AI}^{[3,4,8,9]}$. Blockchain, an emerging technology using a distributed ledger to provide immutable, shared, and transparent records of all operations that happen to the network, will enable a new generation of transactional applications that establish trust, accountability, and transparency. Introducing the blockchain technology into medical service platforms based on AI will effectively carry out the in-depth learning and research, realize the safe, unchangeable, and traceable transmission of information as well as transmission of the diagnostic model, and improve the transparency and credibility of the algorithm diagnostic report. With the management of chronic diseases based on multi-center data collection and distributed storage, patients may reduce their demand for more complex interventions, which in turn will reduce the number of hospitalizations, the burden of medical examination, and expenses after acute attacks. In summary, the combination of sensorbased medical data and intelligent algorithms as well as storage and sharing through blockchain technology will help to improve the management of chronic diseases by integrating physiological and clinical data in real-time ${ }^{[9-11]}$.

1 TRADITIONAL PREVENTION AND TREATMENT STRATEGIES FOR CHRONIC DISEASES

The traditional chronic disease management model is based on the process of "diagnosis, treatment, rehabilitation, and follow-up". At present, the modes of chronic disease management mainly include the chronic care mode, chronic disease self-management mode, and transitional care mode ${ }^{[1,3,8-10]}$. However, there are some related problems in the current chronic disease management, such as a short management radius, high manual dependence, less developed informationization, poor intelligence, blurred responsibility, and privacy concerns. Patients have neither a good experience nor a sense of access, which causes people to be unwilling to undertake chronic disease management ${ }^{[11]}$. Moreover, due to the lack of long-term dynamic monitoring data, doctors often fail to follow up with the patients in a timely manner, leading to diagnosis failure and an unsynchronized treatment process.

\section{MEDICALAI BASED ON WEARABLE DEVICES}

Wearable devices, video devices, and other human internet-of-things devices add end intelligence to effectively improve the proactivity, timeliness, and stability of the collection of data. For example, they provide local intelligence services for endpoint devices using field programmable gate array capsulation ${ }^{[4,5,12]}$. With the rapid development of $5 \mathrm{G}$ and fog-end computing nodes, they are used in the vicinity of patients, reducing the loss in data transmission. The application of cloud intelligence mainly focuses on the training of massive samples, while the immediate deployment of fog and edge devices is bringing computer capacity close to the deployed processor, which can be used for model inference ${ }^{[13]}$. According to the collected patient health information, medical services based on AI can generate digital twin models that accurately express a patient's personal health characteristics and timely judge a patient's current physical health status to assist with the management of chronic disease ${ }^{[14]}$. Medical diagnosis methods by AI mainly include big data mining, rule engines, deep learning, and so on. Big data mining extracts the association among a humongous quantity of data to retrieve health management models for chronic disease diagnosis. A rule engine executes business-related rules based on expert knowledge to make decisions on a patient's health status. With the increasing amount of available healthcare data and the rapid evolution of AI, deep learning constructs a multi-layer complex structure or multiple processing layers composed of multiple nonlinear transformations for processing multimodal data to combine low-level features to form a more abstract, structured, high-level representation of a patient's healthcare data for diagnosis ${ }^{[15]}$. AI-based wearable devices automatically process chronic disease health data timely and effectively, thus achieving the effect of auxiliary diagnosis with limited medical 
resources.

The methods and equipments for the collection of most healthcare data are relatively mature for the management of chronic diseases, such as blood pressure data, fasting glucose, heart rate, and pulse ${ }^{[16]}$. Presently, pose estimation is an important research field that is related to the diagnosis of chronic diseases such as lung-related disease and heart-related disease. The data of attitude and recognition have a high comprehensive value. Currently, the collection of attitude data mainly includes electromyogram and skin electronic wearable sensing devices, wearable visual positioning identifiers, and methods of computer vision-based bone key location.

\section{INTEGRATION OF AI, BLOCKCHAIN, AND WEARABLE TECHNOLOGY FOR THE MANA- GEMENT OF CHRONIC DISEASES}

Currently, the management of chronic diseases involves the passive treatment of disease and the active monitoring of the health status. The emergence of a series of cutting-edge technologies such as AI, blockchain, and wearable technology has led to some new ideas for the real-time monitoring of chronic diseases $^{[1-3,6,8,10,15]}$. The previous methods of inspection for physiological data occurred in the hospital. The management of chronic diseases requires patient monitoring, status assessment, and recommendations. Presently, the frequent collection of personal physical and health data has been greatly improved with decentralized wearables, digital equipment, and mobile applications. The use of wearables can record the healthcare data of patients ubiquitously ${ }^{[16,17]}$, while AI can assist in increasing the efficiency of healthcare delivery. With the application of AI, the healthcare industry will be changed; it will gather the experience of doctors, pharmacists, and nutritionists to provide comprehensive, continuous, and active management for patients with chronic diseases in order to achieve a scientific management mode that promotes the development of the healthy state, delays disease progression, and reduces the disability rate ${ }^{[18]}$. With the assistance of machine learning, the intelligent medical unit can analyze large volumes of health data to establish complex, nonlinear relationships between our body and diseases that cannot easily be expressed in the form of an equation, enabling greater accuracy in the outcome ${ }^{[19]}$. By exploring AI methods in combination with wearable technology, it will provide better services for patients. However, the crucial issue in the management of chronic diseases is to collect and analyze patients' physiological parameters of healthcare data in a timely manner while protecting patient privacy $^{[20]}$. The transmission of personal health data between medical units is often limited by restrictions due to privacy and data security. Additionally, the transparency and testing validation for many models and published deep learning systems are lacking. Acting as a shared ledger for data management in a decentralized, secure, and trusted manner, blockchain technology could be a potential solution to this problem and provide traceability and auditability of health data ${ }^{[20,21]}$. The blockchain system leverages smart contracts to control the co-chain data of wearable devices to protect a patient's privacy and realizes cross-chain information interaction and value transfer of different blockchain platforms through a unified consensus plugin ${ }^{[22]}$. The integration of deep learning, wearables, and blockchain technology will help to improve efficiency automation, data security, and privacy as well as enhance the quality of management of chronic diseases ${ }^{[23-26]}$. Fig. 1 shows a hypothetical technical process in which AI and blockchain can learn the data from wearable devices and upload it to a healthy cloud platform or a special data management structure unit to continuously improve the relevant algorithm model.

At present, research is mainly based on the major problems and challenges of chronic disease prevention and treatment. The data of patients can be collected through wearable devices and shared to medical institutions reliably with the help of blockchain privacy protection and reliable data backtracking ${ }^{[27]}$. Medical institutions can handle a large amount of chronic disease information with the help of AI technology. The wearable devices provide important technical support for health digital twins, ensure the wireless synchronous calculation speed of multiple wearable sensors, effectively solve the overall calculation problem of multi-point detection health, and complete the multimodal calculation of blood glucose, blood pressure, heart rate, blood oxygen content, temperature, respiration, and posture through an adaptive protocol ${ }^{[10-12,19,25,26]}$. An overview of the information processing is shown in fig. 2.

Focusing on the main challenges in the management of common chronic diseases, we proposed a theoretical system to collect health data of chronic diseases through wearable devices and intelligent terminal devices. Based on blockchain technology, we designed the system to protect patient privacy as well as to share and trace patient data. In addition, we summarized the theoretical feasibility and current trends and methods as well as launched the topic of ethical issues. The framework of our conception of an integrated system for chronic disease management is depicted in fig. 3 .

In this review, the integration and the theoretical principles of AI, blockchain, and wearable devices in the management of chronic disease data are presented. Moreover, we demonstrate the practical application of these technologies in the prevention and treatment of four typical chronic diseases. Finally, the shortcomings 


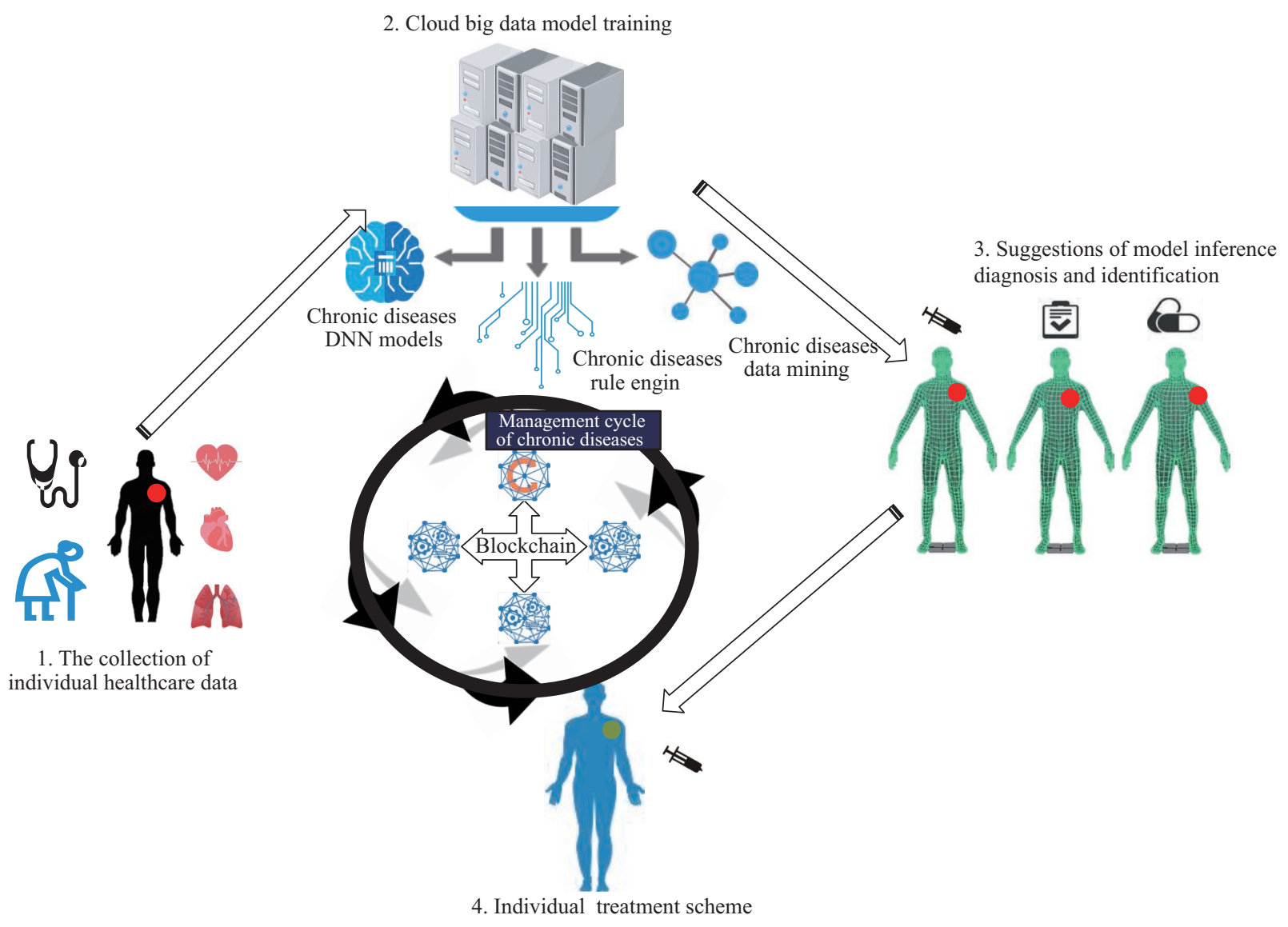

Fig. 1 The technical process of chronic disease management based on AI and blockchain technology

of this new paradigm and future research directions are discussed.

\section{CASE STUDIES IN THE MONITORING AND MANAGEMENT OF CHRONIC DISEASES}

\subsection{Overview}

In the management of chronic disease, European and North American countries have accumulated a lot of experience. However, comprehensive and systematic solutions for chronic disease management are lacking in many developing countries. Seniors are affected by numerous chronic conditions, such as diabetes, hypertension, osteoarthritis, osteoporosis, cardiovascular diseases, and cerebrovascular diseases ${ }^{[18]}$. This new era has given rise to new methods of disease diagnosis and treatment. The continuous development of big data, AI, blockchain, and wearable devices has brought medicine into the digital age ${ }^{[19,26,28]}$. In this series of digital connections, the combination of these technologies will give full play to its advantages. In the context of long-term continuous health data collection, we combine wearables with AI, try to explore a new method of chronic disease management, and introduce blockchain technology to this comprehensive healthcare system. To demonstrate the principle and structure in detail, we mainly discuss the typical management of four chronic diseases: cardiovascular disease, pulmonary disease, diabetes mellitus, and chronic diseases requiring sports rehabilitation.

\subsection{Cardiovascular Diseases}

In recent years, cardiovascular disease has become a serious and harmful problem that threatens global health and affects human life $\mathrm{e}^{[27,28]}$. For example, hypertension is a chronic cardiovascular disease with a high incidence that can lead to stroke, heart failure, renal failure, and other severe diseases. The early discovery, the effective control of the risk factors, and the establishment of an early warning model have an important significance on the outcome of cardiovascular disease ${ }^{[29]}$.

In the past few years, the use of AI in the diagnosis and treatment of cardiovascular disease has grown, which has improved the quality of care, cost-effectiveness, and mortality in patients with heterogeneous diseases, such as pulmonary hypertension, coronary artery disease, heart failure, and cardiomyopathy ${ }^{[30]}$. AI enables continuous real-time monitoring, whether in the home or medical settings and has built-in methods of care that can be upgraded and intervened upon demand, ultimately enabling patients to self-manage their condition and to improve their prognosis. From the theoretical perspective of the algorithm, previous studies mainly use naive Bayesian, 


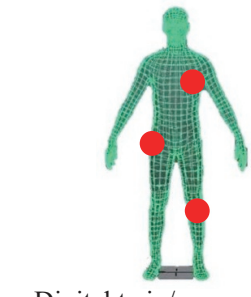

Digital twin/ Auxiliary diagnosis

0

Wearable/Vision devices

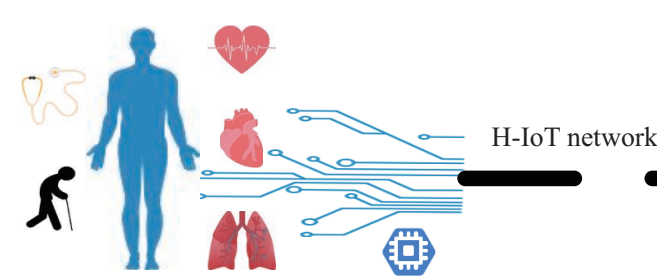

Endpoint intelligence rision
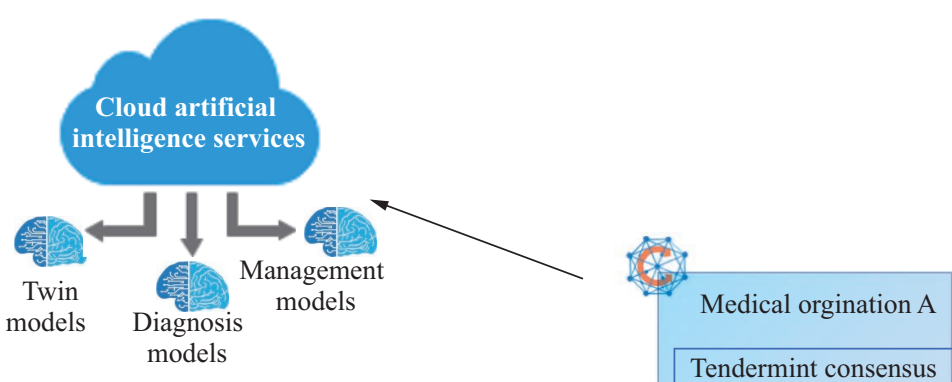

Tendermint consensus

Fig. 2 The workflow of information processing

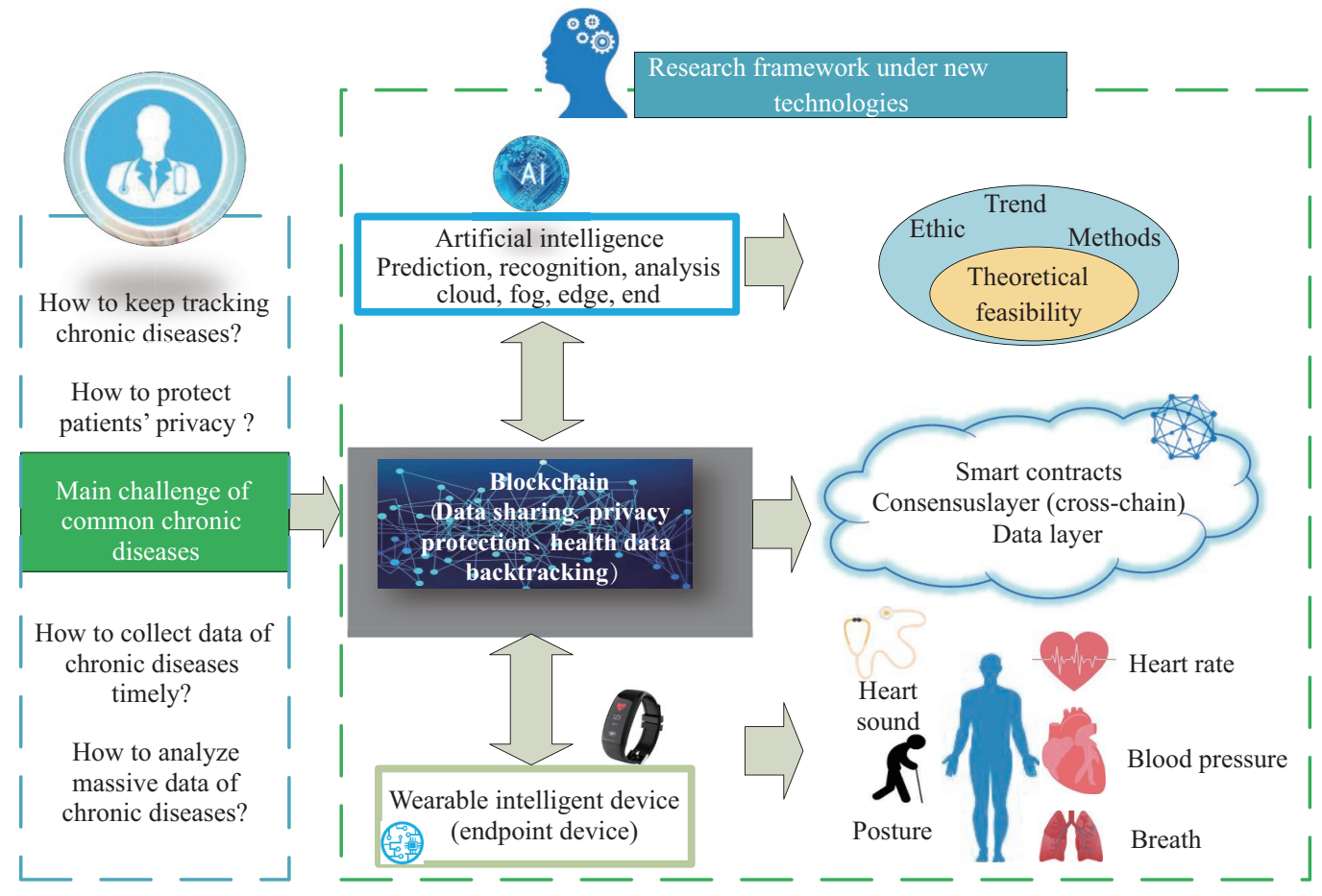

Fig. 3 The conception of the integrated system for chronic disease management

logistic regression, and support vector machine classification model algorithms to respectively build a risk model for chronic and critical illness events caused by cardiovascular function loss. Because the majority of 12-lead electrocardiogram (ECG) machines can provide analysis and enable the provisional diagnosis of myocardial infarction and cardiac arrhythmia, the AI algorithm for detecting ST elevation is commonly available ${ }^{[27,28]}$. In a study by Kaspar et $a l^{[27]}$, they found that wearable cardioverter defibrillators with
AI technology could protect patients from sudden cardiac arrest during nonhospitalization until the implantable cardioverter-defibrillator was re-implanted. By using wearable fabrics, Tsukada et al ${ }^{[28]}$ developed a sport-vest polymer made of nanofibers coated with a conductive coating, which made the ECG electrodes closely contact with the human body. With the help of this signal, the related application can display the ECG and collect health data in real-time, thus improving the comfort and avoiding the risk of allergy. In addition, 
related physiological data are uploaded to the cloud for analysis by professional doctors, enabling remote monitoring of heart disease.

Moreover, combined with the widespread availability of broadband technology, the rapid development and low cost of hand-held echocardiography devices have brought new opportunities for screening and early diagnosis of valvular heart disease in the community. Once images have been acquired, the AI-based system can accelerate appropriate intervention in valvular heart disease, and the automated alerts from an integrated hand-held echocardiogram device with electronic sensors can be delivered directly to healthcare providers ${ }^{[29,30]}$. This form of convenient data relay can significantly improve the timeliness of clinical treatment. In treatment planning, as recommended by the guidelines, the most appropriate treatment should be determined by a multidisciplinary heart team, usually taking into account patient comorbidities, age, degree of weakness, degree of independence, anatomic conditions, preoperative mortality, and patient preferences ${ }^{[31]}$. Clinical decision support systems based on AI have been proven to assist preoperative planning by incorporating imaging data from echocardiography or computed tomography into algorithms that help to determine the appropriate valve size (and even the valve type) for interventional therapy in a fast and reliable manner ${ }^{[28-31]}$.

In some cases, machine learning often encounters bottlenecks due to the difficulty of obtaining sufficient physiological health data. The challenge is to compile health information from diverse sources, such as wearable devices, portable ECG monitors, existing hospital-based electronic medical records, biobanks, and nonmedical data from social media and activity $\log 5^{[30]}$. Based on blockchain technology, a natural foundation can be formed to standardize the health data structure for AI training, clinical trials, and supervision. When combined with AI and blockchain technology, the diagnosis and treatment of cardiovascular diseases can be divided into two parts. The first part includes the establishment of a distributed storage database and data cleaning in a specific time through in-depth learning to integrate the scattered information and fully tap the potential of data by making the software model learn the medical data history. The second part is to make a comparative analysis and to evaluate the intelligent algorithm model in cardiovascular disease $^{[31]}$. Blockchain technology provides the capacity to trace independent block (record) types and dissect granular data ${ }^{[25,30,31]}$. By integrating blockchain and AI, the complementary advantages can be achieved at the technical level, which can promote the quality of personalized cardiovascular treatment by greatly improving the availability of data for AI development and training, sharing proprietary algorithms for promotion, decentralizing databases of different providers or medical systems, and motivating solutions that improve results compared to other providers or medical systems ${ }^{[32]}$. Based on the integrated system, the management of cardiovascular diseases will be efficient and user-friendly, and the knowledge of patients will be significantly improved. Therefore, the adherence of drug use and efficiency of anticoagulants will be enhanced, and the risk of a malignant heart attack may be gradually reduced.

\subsection{Pulmonary Diseases}

Chronic pulmonary diseases mainly include chronic bronchitis, pulmonary tuberculosis, chronic obstructive pulmonary disease (COPD), and interstitial pulmonary diseas $\mathrm{e}^{[33-35]}$. Common respiratory disorder diseases have a significant morbidity and mortality, and approximately 210 million people are affected around the world; therefore, chronic pulmonary diseases have become a serious health problem for the elderly ${ }^{[33]}$. For these patients, continuous monitoring of their disease progression and physical health status is essential for controlling their disease. Various wearable sensors have been developed to monitor the physical activities of patients with COPD. The use of wearable devices allows uninterrupted assessment of lung conditions in the home, including pulmonary breath sounds, oxygen saturation, heart rate, pulse, and quality indicators that detect activity and airborne aerosols associated with lung disease. To evaluate the staged effect of clinical treatment, Colantonio et a ${ }^{[34]}$ used wearable devices and built a wireless sensor network system to monitor blood oxygen saturation, ECG, and the sound and rate of respiration in patients.

Additionally, AI has shown great medical prospects in the research of pulmonary disease ${ }^{[33,34]}$. A digital diagnostic model based on an AI algorithm can give an accurate assessment of several lung conditions such as chronic obstructive lung disease, pneumonia, and chronic asthma. For example, an artificial neural network developed by Bugajski et al ${ }^{[35]}$ shows great validity in predicting self-management among patients with COPD through understanding and reporting of patient symptoms, and the accuracy was found to be $93.8 \%$. Similarly, by using a total of 22 variables to predict the initial diagnosis of adult asthma, Tomita et $a l^{[36]}$ explored the effectiveness of deep neural networks in modeling combinations of symptoms, physical signs, and objective tests, thus demonstrating the feasibility of the conception. In addition, Ather et al ${ }^{[37]}$ carried out volumetric segmentation of 7927 nodules from the National Lung Screening Trial cohort in the United States via a deep-learning model that was initiated with a single click point inside the nodule, showing that AI can be used in the detection, classification, and follow-up of pulmonary nodules. The related mobile applications also have been implemented in 
the management of pulmonary diseases; for instance, Res-App ${ }^{[38]}$ is an applet in mobile devices that is used for monitoring breathing through the microphone and providing a personalized evaluation for lower respiratory tract disease, asthma, pneumonia, and bronchiolitis. Moreover, in the management of chronic pulmonary diseases in children, Yu et al ${ }^{[39]}$ compared four machine learning-based models, including naive Bayes, support vector machines, logistic regression, and CatBoost, and proposed that the AI model can quickly and accurately identify children with asthma; therefore, it will help patients receive early intervention in the case of an emergency. Another example using AI is for the treatment of obstructive sleep apnea, which is a pulmonary disease often associated with obesity and has comorbidities including arrhythmias, hypertension, stroke, and coronary heart disease ${ }^{[40]}$. In the blockchain and AI-based system, information can be obtained from the nonintrusive collection of reallife data concerning daily activities via smartphones and connected wearable devices. A wide spectrum of physiological signals generated during home settings can be captured via the wireless network and interpreted by cloud-based machine-learning-derived algorithms ${ }^{[41]}$. Approaches including multimodal remote telemonitoring and telemedicine, smartphone engagement tools, and internet-based management platforms need to be supported by Big Data analysis and $\mathrm{AI}^{[38-41]}$. The integration of decentralized multimodal data and algorithms will enhance the capability of caregivers to make prompt adjustments and to provide individualized guidance to patients with obstructive sleep apnea.

\subsection{Diabetes Mellitus}

With the rapid development of the economy and changes in diet and lifestyle, the incidence of diabetes is increasing throughout the world ${ }^{[42]}$. As a serious chronic disease, diabetes is defined as the impaired regulation of carbohydrate metabolism and abnormal glucose levels in the blood ${ }^{[43]}$. The absence of proper care will significantly induce the undulation of blood glucose concentrations in diabetics throughout the day, which may lead to adverse consequences such as renal failure, atherosclerosis, diabetic retinopathy, high blood pressure, and coma ${ }^{[43,44]}$. The control of blood glucose levels is essential for diabetic patients. Improving the ability of self-monitoring for diabetics is necessary to reduce morbidity and mortality. The conventional method for monitoring blood glucose levels is drawing finger-prick blood samples directly and analyzing them by a biochemical analyzer, which is an invasive and inconvenient process.

With the rapid development of biosensors and wireless communication technologies, a new generation of noninvasive wearable blood glucose monitoring devices has emerged. Recently, a wearable artificial pancreas composed of a flexible core system as a brain and three wedges has become commercially available for glucose level monitoring and regulation. Another wearable device designed to measure blood sugar levels in patients with diabetes is smart contact lenses, which were developed by Google/Life Sciences (USA) in collaboration with Novartis (Basel, Switzerland). In addition, wearable technology based on AI has been developed for the real-time monitoring of blood glucose levels of diabetic patients and automatic regulation of blood glucose by controlling insulin pumps. AI is of great significance for the personalized management of patients with diabetes, including formulating scientific diet plans, conducting the appropriate physical exercise, tracking the occurrence of common complications, and even providing technical support for the use of insulin. To realize self-management of blood glucose levels, Fernández-Caramés et $a{ }^{[42]}$ designed a system that enhances continuous monitoring of glucose by adding wearable capabilities to allow for monitoring remotely and alerting patients about potential emergencies. In addition, an incentive system based on blockchain named GlucoCoin was devised to motivate users to add new data to the system.

Among the complications of diabetes, diabetic retinopathy is common and may cause irreversible blindness. Early intervention of diabetic retinopathy can significantly improve the prognosis of patients and their quality of life. With the help of AI, Hao et $a l^{[43]}$ examined diabetic retinopathy screening and found that the screening rate of diabetic retinopathy in an outpatient clinic increased from $28.4 \%$ to $81.3 \%$. In summary, the management of blood glucose levels using AI and wearable devices is a safe and effective method, which can effectively reduce the occurrence of diabetic complications such as retinopathy as well as diabetic foot problems ${ }^{[25,26,44]}$. Information integration systems based on AI and blockchain technology can make a risk assessment and timely warning for diabetic ketoacidosis, hypoglycemia, and hyperglycemic hyperosmolar syndrome, which will greatly enhance the safety of patients with diabetes mellitus ${ }^{[42,45]}$.

\subsection{Chronic Diseases Requiring Sports Rehabili- tation}

In the recovery process of chronic diseases of the motor system, the related data of gait, balance, and coordination are important; however, it is difficult to accurately assess these indexes ${ }^{[19,21,22,24,46,47]}$. Physiologically, patient data can be collected comprehensively by tracking the location of bones based on wearables, thus providing perfect data for remote rehabilitation. Wearable systems based on blockchain and AI are capable of monitoring posture and providing real-time feedback alerts for patients with a persistently abnormal posture to correct their posture, thereby reducing the total time in 
poor posture conditions; this technology has a great application potential in physical rehabilitation ${ }^{[48]}$. It integrates cutting-edge models in the fields of medical rehabilitation theory, wearable posture analysis, health data twinning, AI, three-dimensional reconstruction, and machine learning; and it provides data support and guarantees for remote rehabilitation follow-up. According to the tracking equipment worn by the patient, the patient's three-dimensional image can be reconstructed by AI. The system can combine a health warning and an abnormal analysis of heart rate, pulse, respiration, stride, pace, blood oxygen, step frequency, upper limbs, waist, and lower limbs, according to the patient's rehabilitation movement ${ }^{[49-51]}$.

Gait disturbance is an important sign of compressive myelopathy. At present, the rehabilitation of lower limbs is mainly focused on gait training ${ }^{[46,48,51]}$. Gait can be monitored in real-time by sensors installed on shoes or wearable devices. It is possible to promptly diagnose the progression of myelopathy in patients with cervical stenosis or ossification of a posterior longitudinal ligament. Hsu et $a l^{[47]}$ adopted multiplacement wearable sensors to analyze and classify the gait features of patients with nervous system diseases and to guide the selection of rehabilitation exercise programs. Furthermore, it is possible to develop a wearable monitoring system in which paralysis or deterioration of myelopathy can be detected early in patients with spinal diseases using $\mathrm{AI}^{[48]}$. Additionally, Merali et al ${ }^{[51]}$ demonstrated the application of the machine learning approach to predict outcomes after surgery for degenerative cervical myelopathy. The management of spinal posture is important for patients with scoliosis or chronic injury, and some wearables that can manage the posture of the spine are already commercially available. With the help of wearable devices, the research on measuring the physiological curvature and posture of the spine in patients with chronic diseases of the motor system is conducted continuously. In spinal surgery, it is often necessary to insert implants such as pedicle screws, Kirschner wires, and artificial intervertebral discs into patients to achieve a satisfactory reset effect ${ }^{[49,50]}$. In the future, it will be possible to insert instruments with sensors into related structures of the human body, which can carry out real-time monitoring of physiological states ${ }^{[52]}$. Wearable sensors minimize the potential limitations of traditional assessment tools by generating quantitative data and greatly improving the home monitoring of patients through a timely internet-of-things data transmission mechanism, such as analyzing surface electromyogram data collected during musculoskeletal function to assess activity patterns of abnormal muscles.

In rehabilitation training, some meaningful experimental platforms have been established to prevent some accidents and to increase the effectiveness. In their evidence-based fall prevention plan, Famati et al ${ }^{[53]}$ designed a technical platform that allows all relevant variables to be concentrated in a safe database for monitoring the patient's body position to prevent falling during the period of rehabilitation. In addition, Chae et $a l^{[54]}$ evaluated a network-based machine learning upper limb home rehabilitation system and proposed that the machine learning model can promote participation in family training and improve functional scoring of the Wolf Motor Function Test as well as the shoulder range of motion of flexion and internal rotation during the treatment. Moreover, the application of wearable devices in the rehabilitation of patients after a sports injury, especially their combination with virtual reality, augmented reality, and mixed reality technology, will contribute to evaluate the effect of sports rehabilitation, to make the training more interesting, and to improve the patient's compliance ${ }^{[36,55]}$. Therefore, we believe that it is imperative that a sophisticated system of sports rehabilitation will be established based on wearables and blockchain technology, which will potentially reduce the necessity for more complex interventions and in turn reduce the probability of secondary traumas in the patients ${ }^{[1,2,8,18,19,35,55,56]}$.

\section{DISCUSSION}

\subsection{Principal Findings}

The combination of AI, blockchain, sensors, and wearable devices for monitoring body activity, various environments, and location data will improve the efficiency of implementing chronic disease prevention; this technology has shown great commercial and medical prospects $^{[19,20,26,29,35,42,47,51,57,58]}$. However, making full use of such scattered real-time multidimensional data requires the establishment of structured and intelligent multidisciplinary integration as well as the iteration and innovation of machine learning and AI for the high-performance calculation of data ${ }^{[54]}$. A digital chronic disease management platform based on AI, blockchain, and wearable devices can effectively utilize the advantages of these emerging technologies, regularly send drug intake reminders to patients, process the collected monitoring data in real-time, and issue abnormal data warnings to patients and doctors, thus realizing remote monitoring of the treatment process $^{[38]}$. For elderly patients in remote areas, when they are far away from relevant hospitals or when it is inconvenient to use medical facilities, such as during a pandemic, remote telemedicine has shown great value in the control of chronic diseases ${ }^{[18,19,25,58]}$. With the development of wireless communication networks, blockchain, wearable sensors, and AI technology, the diversity and accuracy of the medical internet of things are expected to be greatly improved ${ }^{[20,31,32,37,39,43,50,59]}$. 
The inherent value of these technologies can be transformed into clinical and social usefulness based on the ideas and creativity of clinicians who treat patients.

Although the application of these technologies in the medical field has improved people's well-being, they are also prone to causing ethical risks ${ }^{[32,33,39,58,59]}$. First of all, the AI algorithm used for disease diagnosis may have problems of security and accuracy, which may cause harm to a patient's health. Second, the interpretability of AI may conflict with the patient's basic right to know. In addition, a fair price mechanism for AI diagnostics may harm the public interest. AI mimics the decision-making process of the human brain; it is not true human intelligence but weak AI. The patient data are collected according to the needs of the algorithm design, while the algorithm and the rules themselves are provided by humans. As a consequence, ethical and moral constraints are still human constraints, existing AI methods are still tools, and the core of ethical review is still the maker of AI itself. Moreover, the anti-interference ability and security threat of transmission technology have become prominent problems in the medical internet of things. From the perspective of connected devices, the wearable devices are forced to compete with the connection between Wi-Fi and non-Wi-Fi devices running at the same frequency, which leads to the possibility of network connection interruption and the problem of alarm transmission $\left.{ }^{[57,} 58,60\right]$. Furthermore, when implementing blockchain in the structure of this system, there will be some problems to be solved, such as interoperability issues, efficiency issues, and security issues. When encountering a problem requiring cooperation, blockchain-based service providers and users must connect seamlessly, but the standards among different institutions are not unified. The general standard of blockchain will accelerate the industry to reach an agreement on blockchain and contribute to the formation of a largescale ecosystem of a social blockchain. With the amount of data growing exponentially, the blockchain database has higher requirements for network speed, and more reasonable frameworks need to be designed to avoid blockchain efficiency problems across sectors ${ }^{[59]}$. Of note, $51 \%$ of malicious attacks are security issues that must be considered ${ }^{[60]}$. Last but not the least, the issue of related regulatory policies must be addressed. Regulatory policies are important factors for the stable development of these technologies. Therefore, related policies need to be introduced systematically as soon as possible.

\subsection{Limitations}

The results of this study must be interpreted with caution due to multiple limitations. First, the results of this review are primarily for medical scenarios and relevant units and do not apply to other areas such as finance and marketing. Second, the content is from the fields of medical, informatics, AI, and computer science, and the professional perspectives may be limited to science and engineering. Policy and ethical studies may be inappropriate. In addition, this review was limited to studies reported in English, while some studies that are reported in other fields and languages may have been overlooked. Finally, the application scenarios of AI based on blockchain and wearable devices in the medical field are diverse, and we did not show all aspects of its principles and framework in detail.

\section{CONCLUSIONS}

This review describes the innovative integration of blockchain and AI-based wearable devices in the management of chronic diseases. Several techniques for monitoring patients were presented. The shortcomings of this integrated system were also demonstrated when discussing and recommending possible digital solutions. We also highlighted the significant issues and challenges in the clinical and daily management of chronic diseases. In the future, it is necessary to give priority to the use of patient big data in the telemedicine environment, and it is recommended to implement blockchain technology to establish a medical management system for chronic diseases. Furthermore, sources of data on the environment and posture of the human body, such as real-time location, weather conditions, video materials, sounds, and builtin sensors, need to be added to facilitate the provision of appropriate emergency assistance in the case of acute exacerbation. Overall, the integration of AI, wearable devices, and blockchain is likely to play a key role in the management of chronic diseases, and it will be exciting to see how the use of this technology benefits patients and doctors in everyday healthcare.

\section{Conflict of Interest Statement} interest.

The authors declare that they have no conflicts of

\section{REFERENCES}

1 Bauer UE, Briss PA, Goodman RA, et al. Prevention of chronic disease in the 21st century: elimination of the leading preventable causes of premature death and disability in the USA. Lancet, 2014,384(9937):45-52

2 Bashshur RL, Shannon GW, Smith BR, et al. The empirical foundations of telemedicine interventions for chronic disease management. Telemed J E Health, 2014, 20(9):769-800

3 Allegrante JP, Wells MT, Peterson JC, et al. Interventions to Support Behavioral Self-Management of Chronic Diseases. Annu Rev Public Health, 2019,40:127-146

4 Katwa U, Rivera E. Asthma Management in the Era of Smart-Medicine: Devices, Gadgets, Apps and Telemedicine. Indian J Pediatr, 2018,85(9):757-762 
5 Hamine S, Gerth-Guyette E, Faulx D, et al. Impact of mHealth chronic disease management on treatment adherence and patient outcomes: a systematic review. J Med Internet Res, 2015,17(2):e52

6 Subramanian M, Wojtusciszyn A, Favre L, et al. Precision medicine in the era of artificial intelligence: implications in chronic disease management. J Transl Med, 2020,18(1):472

7 Contreras I, Vehi J. Artificial Intelligence for Diabetes Management and Decision Support: Literature Review. J Med Internet Res, 2018,20(5):e10775

8 Buekers J, Theunis J, De Boever P, et al. Wearable Finger Pulse Oximetry for Continuous Oxygen Saturation Measurements During Daily Home Routines of Patients With Chronic Obstructive Pulmonary Disease (COPD) Over One Week: Observational Study. JMIR Mhealth Uhealth, 2019,7(6):e12866

9 Mekov E, Miravitlles M, Petkov R, et al. Artificial intelligence and machine learning in respiratory medicine. Expert Rev Respir Med, 2020,14(6):559-564

10 Song Y, Min J, Gao W, et al. Wearable and Implantable Electronics: Moving toward Precision Therapy. ACS Nano, 2019,13(11):12280-12286

11 Cheung CC, Krahn AD, Andrade JG, et al. The Emerging Role of Wearable Technologies in Detection of Arrhythmia. Can J Cardiol, 2018,34(8):1083-1087

12 Guo Y, Liu X, Chen W, et al. A review of wearable and unobtrusive sensing technologies for chronic disease management. Comput Biol Med, 2021,129:104163

13 Lin LF, Lin YJ, Lin YH, et al. Feasibility and efficacy of wearable devices for upper limb rehabilitation in patients with chronic stroke: a randomized controlled pilot study. Eur J Phys Rehabil Med, 2018,54(3):388396

14 Pilozzi A, Huang X. Overcoming Alzheimer's Disease Stigma by Leveraging Artificial Intelligence and Blockchain Technologies. Brain Sci, 2020,10(3):183

15 KuoTT, GabrielRA, Ohno-MachadoL, etal.EXpectation Propagation LOgistic REgRession on permissioned blockCHAIN (ExplorerChain): decentralized online healthcare/genomics predictive model learning. J Am Med Inform Assoc, 2020,27(5):747-756

16 M Bublitz F, Oetomo A, P Morita P, et al. Disruptive Technologies for Environment and Health Research: An Overview of Artificial Intelligence, Blockchain, and Internet of Things. Int J Environ Res Public Health, 2019,16(20):3847

17 Peyvandi A, Majidi B, Patra J, et al. Computer-AidedDiagnosis as a Service on Decentralized Medical Cloud for Efficient and Rapid Emergency Response Intelligence. New Gener Comput, 2021,27:1-24

18 Silva P, Jacobs D, Neal G, et al. Implementation of Pharmacogenomics and Artificial Intelligence Tools for Chronic Disease Management in Primary Care Setting. J Pers Med, 2021,11(6):443

19 Lu L, Zhang J, Ye Z, et al. Wearable Health Devices in Health Care: Narrative Systematic Review. JMIR Mhealth Uhealth, 2020,8(11):e18907

20 Jiang W, Majumder S, Monday T, et al. A Wearable Tele-Health System towards Monitoring COVID-19 and Chronic Diseases. IEEE Rev Biomed Eng, 2021,1:1

21 Dwivedi AD, Srivastava G, Singh R, et al. A
Decentralized Privacy-Preserving Healthcare Blockchain for IoT. Sensors (Basel), 2019,19(2):326

22 Kalid N, Zaidan AA, Muzammil H, et al. Based Real Time Remote Health Monitoring Systems: A Review on Patients Prioritization and Related "Big Data" Using Body Sensors information and Communication Technology. J Med Syst, 2017,42(2):30

23 Qadri YA, Nauman A, Kim SW, et al. The Future of Healthcare Internet of Things: A Survey of Emerging Technologies. IEEE Communications Surveys \& Tutorials, 2020,22(2):1121-1167

24 Koydemir HC, Ozcan A. Wearable and Implantable Sensors for Biomedical Applications. Annu Rev Anal Chem (Palo Alto Calif), 2018,12,11(1):127-146

25 Xie Y, Zhang J, Wang $\mathrm{H}$, et al. Applications of Blockchain in the Medical Field: Narrative Review. J Med Internet Res, 2021,23(10):e28613

26 Zheng X, Sun S, Ordieres-Meré J, et al. Accelerating Health Data Sharing: A Solution Based on the Internet of Things and Distributed Ledger Technologies. J Med Internet Res, 2019,21(6):e13583

27 Kaspar G, Sanam K, Gholkar G, et al. Long-term use of the wearable cardioverter defibrillator in patients with explanted ICD. Int J Cardiol, 2018,272(1):179-184

28 Tsukada YT, Tokita M, Iwasaki Y, et al. Validation of wearable textile electrodes for ECG monitoring. Heart Vessels, 2019,34(7):1203-1211

29 Abe Y, Ito M, T anaka C, et al. A novel and simple method using pocket-sized echocardiography to screen for aortic stenosis. J Am Soc Echocardiogr, 2013,26:589596

30 Thoenes M, Agarwal A, Grundmann D, et al. Narrative review of the role of artificial intelligence to improve aortic valve disease management. J Thorac Dis, 2021, 13(1):396-404

31 Barrett M, Boyne J, De Wit K, et al. Artificial intelligence supported patient self-care in chronic heart failure: a paradigm shift from reactive to predictive, preventive and personalised care. EPMA J, 2019,10(4):445-64

32 Fan X, Yao Q, Li Y, et al. Multiscaled Fusion of Deep Convolutional Neural Networks for Screening Atrial Fibrillation From Single Lead Short ECG Recordings. IEEE J Biomed Health Inform, 2018,22(6):1744-1753

33 Kaplan A, Cao H, Kocks JWH, et al. Artificial Intelligence/Machine Learning in Respiratory Medicine and Potential Role in Asthma and COPD Diagnosis. J Allergy Clin Immunol Pract, 2021,9(6):2255-2261

34 Colantonio S, Govoni L, Vitacca M, et al. Decision Making Concepts for the Remote, Personalized Evaluation of COPD Patients' Health Status. Methods Inf Med, 2015,54(3):240-247

35 Bugajski A, Lengerich A, Szalacha L, et al. Utilizing an Artificial Neural Network to Predict Self-Management in Patients With Chronic Obstructive Pulmonary Disease: An Exploratory Analysis. J Nurs Scholarsh, 2021,53(1):16-24

36 Tomita K, Nagao R, Tohda Y, et al. Deep learning facilitates the diagnosis of adult asthma. Allergol Int, 2019,68(4):456-461

37 Ather S, Kadir T, Gleeson F. Artificial intelligence and radiomics in pulmonary nodule management: current status and future applications. Clin Radiol, 
2020,75(1):13-19

38 Porter P, Abeyratne U, Della P, et al. A prospective multicentre study testing the diagnostic accuracy of an automated cough sound centred analytic system for the identification of common respiratory disorders in children. Respir Res, 2019,20(1):81

$39 \mathrm{Yu} \mathrm{G}, \mathrm{Li} \mathrm{Z}$, Li S, et al. The role of artificial intelligence in identifying asthma in pediatric inpatient setting. Ann Transl Med, 2020,8(21):1367

40 Pépin JL, Bailly S, Tamisier R, et al. Big Data in sleep apnoea: Opportunities and challenges. Respirology, 2020,25(5):486-494

$41 \mathrm{Wu}$ CT, Li GH, Chien JY, et al. Acute Exacerbation of a Chronic Obstructive Pulmonary Disease Prediction System Using Wearable Device Data, Machine Learning, and Deep Learning: Development and Cohort Study. JMIR Mhealth Uhealth, 2021,9(5):e22591

42 Fernández-Caramés TM, Froiz-Míguez I, Blanco-Novoa $\mathrm{O}$, et al. Enabling the Internet of Mobile Crowdsourcing Health Things: A Mobile Fog Computing, Blockchain and IoT Based Continuous Glucose Monitoring System for Diabetes Mellitus Research and Care. Sensors (Basel), 2019,19(15):3319

43 Hao Z, Cui S, Zhu Y, et al. Application of non-mydriatic fundus examination and artificial intelligence to promote the screening of diabetic retinopathy in the endocrine clinic: an observational study of T2DM patients in Tianjin, China. Ther Adv Chronic Dis, 2020,11:2040622320942415

44 Mendes-Soares H, Raveh-Sadka T, Cohen Y, et al. Assessment of a Personalized Approach to Predicting Postprandial Glycemic Responses to Food Among Individuals Without Diabetes. JAMA Netw Open, 2019, 2(2):e188102

45 Rodriguez-León C, Villalonga C, Munoz-Torres M, et al. Mobile and Wearable Technology for the Monitoring of Diabetes-Related Parameters: Systematic Review. JMIR Mhealth Uhealth, 2021,9(6):e25138

46 Jourdan T, Debs N, Frindel C. The Contribution of Machine Learning in the Validation of Commercial Wearable Sensors for Gait Monitoring in Patients: A Systematic Review. Sensors (Basel), 2021,21(14):4808

47 Hsu WC, Sugiarto T, Lin YJ, et al. Multiple-WearableSensor-Based Gait Classification and Analysis in Patients with Neurological Disorders. Sensors (Basel), 2018,18(10):3397

48 Chomiak T, Xian W, Pei Z, et al. A novel single-sensorbased method for the detection of gait-cycle breakdown and freezing of gait in Parkinson's disease. J Neural Transm (Vienna), 2019,126(8):1029-1036

49 Williamson JR, Telfer B, Mullany R, et al. Detecting
Parkinson's Disease from Wrist-Worn Accelerometry in the U.K. Biobank. Sensors (Basel), 2021,21(6):2047

$50 \mathrm{Nam} \mathrm{KH}, \mathrm{Kim} \mathrm{DH}$, Choi BK, et al. Internet of Things, Digital Biomarker, and Artificial Intelligence in Spine: Current and Future Perspectives. Neurospine, 2019, 16(4):705-711

51 Merali ZG, Witiw CD, Badhiwala JH, et al. Using a machine learning approach to predict outcome after surgery for degenerative cervical myelopathy. PLoS One, 2019,14(4):e0215133

52 Golabchi FN, Sapienza S, Severini G, et al. Assessing aberrant muscle activity patterns via the analysis of surface EMG data collected during a functional evaluation. BMC Musculoskelet Disord, 2019,20(1):13

53 Araújo F, Nogueira MN, Silva J, et al. A TechnologicalBased Platform for Risk Assessment, Detection, and Prevention of Falls Among Home-Dwelling Older Adults: Protocol for a Quasi-Experimental Study. JMIR Res Protoc, 2021,10(8):e25781

54 Chae SH, Kim Y, Lee KS, et al. Development and Clinical Evaluation of a Web-Based Upper Limb Home Rehabilitation System Using a Smartwatch and Machine Learning Model for Chronic Stroke Survivors: Prospective Comparative Study. JMIR Mhealth Uhealth, 2020,8(7):e17216

55 Tropea P, Schlieter H, Sterpi I, et al. Rehabilitation, the Great Absentee of Virtual Coaching in Medical Care: Scoping Review. J Med Internet Res, 2019,21(10): e12805

56 Zhang H, Song C, Rathore AS, et al. mHealth Technologies Towards Parkinson's Disease Detection and Monitoring in Daily Life: A Comprehensive Review. IEEE Rev Biomed Eng, 2021,14:71-81

57 Zhang Y, Yu H, Dong R, et al. Application Prospect of Artificial Intelligence in Rehabilitation and Management of Myasthenia Gravis. Biomed Res Int, 2021,2021:5592472

58 Pareja-Galeano H, Garatachea N, Lucia A. Exercise as a Polypill for Chronic Diseases. Prog Mol Biol Transl Sci, 2015,135:497-526

59 Kiran MPRS, Rajalakshmi P, Bharadwaj K, et al. Adaptive rule engine based IoT enabled remote health care data acquisition and smart transmission system. 2014 IEEE World Forum on Internet of Things (WFIoT), 2014:253-258

60 Tan TE, Anees A, Chen C, et al. Retinal photographbased deep learning algorithms for myopia and a blockchain platform to facilitate artificial intelligence medical research: a retrospective multicohort study. Lancet Digit Health, 2021,3(5):e317-e329

(Received Oct. 27, 2021; accepted Dec. 3, 2021) 